\title{
Rapid Mapping: geomatics role and research opportunities
}

\author{
Andrea Ajmar • Piero Boccardo • Franca Disabato • \\ Fabio Giulio Tonolo
}

Received: 15 October 2014/Accepted: 4 March 2015/Published online: 25 March 2015

(c) The Author(s) 2015. This article is published with open access at Springerlink.com

\begin{abstract}
In recent years an increasing number of extreme meteorological events have been recorded. Geomatics techniques have been historically adopted to support the different phases of the Emergency Management cycle with a main focus on emergency response, initial recovery and preparedness through the acquisition, processing, management and dissemination of geospatial data. In the meantime, the increased availability of geospatial data in terms of reference topographic datasets, made available by authoritative National Mapping Cadastre Agencies or by Collaborative Mapping initiatives like OpenStreetMap, as well as of remotely sensed imagery, poses new challenges to the Geomatics role in defining operational tools and services in support of emergency management activities. This paper is mainly focused on the role of Geomatics in supporting the response phase of the Emergency Management cycle through Rapid Mapping activities, which can be defined as "the on-demand and fast provision (within hours or days) of geospatial information in support of emergency management activities immediately following an emergency event" (source: European Union, http://emergency. copernicus.eu/mapping/ems/service-overview). Management of geospatial datasets (both reference and thematic), Remote Sensing sensors and techniques and spatial
\end{abstract}

Peer reviewed paper presented at conference on Geodesy and Geomatics held at Accademia Nazionale dei Lincei in Rome on June 3, 2014 .

A. Ajmar $\cdot$ F. Disabato $\cdot$ F. Giulio Tonolo $(\square)$

ITHACA, Via P.C. Boggio 61, 10138 Turin, Italy

e-mail: fabio.giuliotonolo@ithaca.polito.it

P. Boccardo

Politecnico di Torino, DIST, Viale Mattioli 39, 10125 Turin,

Italy information science methodologies applied to Rapid Mapping will be described, with the goal to highlight the role that Geomatics is currently playing in this domain. The major technical requirements, constraints and research opportunities of a Rapid Mapping service will be discussed, with a specific focus on: the time constraints of the service, the data quality requirements, the need to provide replicable products, the need for consistent data models, the advantages of data interoperability, the automation of feature extraction procedures to reduce the need for Computer Aided Photo Interpretation, the dissemination strategies.

Keywords Geomatics - Rapid mapping - Emergency response $\cdot$ Remote sensing - Spatial information science

\section{Introduction}

Several disaster statistics and databases can be analyzed to identify the last decades' trends of disaster impacts in terms of economic loss, affected and killed people. Munich Re (2012) highlights increasing trends in the period 2000-2011 both in terms of number of events (more than doubled in the considered period, with a higher occurrence of hydrological and meteorological events) and overall losses, up to an yearly average of approx. 175 bn US\$. Adikari, and Yoshitani (2009) mention that "Trends in natural disasters show they are continuously increasing in most regions of the world in the period 1990-2006. Among all observed natural and anthropogenic adversities, waterrelated disasters are undoubtedly the most recurrent and pose major impediments to the achievement of human security and sustainable socio-economic development". Guha-Sapir et al. (2014), highlight that natural disasters 
reported for 2013 are decreasing (330) when compared to the average annual occurrence from 2003 to 2012 (388). But unfortunately the number of people killed by floods (9819) was the highest of the decade and the number of those killed by storms (8583), the second highest. Most disaster victims in 2013 were sourced by cyclones Hayan, Phailin and Utor/Labuyo (all these emergencies recorded in the Far East), that together affected more than 37 millions of people. From these data, Guha-Sapir et al. (2014) stress that climate change related disasters (floods and hurricanes/cyclones) are more devastating, both in terms of affected people and of economic losses. The United Nations Economic and Social Commission for Asia and the Pacific (UNESCAP 2015) focuses the analysis in Asia and the Pacific regions in 2014, highlighting that "2014 was an atypical year in terms of storms, transboundary floods and landslides, which collectively contributed to the region's total economic losses of US $\$ 59.6$ billion".

Disaster trend analysis is crucial for understanding the patterns of past disasters, and is helpful for examining and perceiving future disaster risk and vulnerability. But taking these data into account, it is also evident the need for operational methodologies, tools and products able to operationally support different phases of the Emergency Management cycle (i.e., "the organization and management of resources and responsibilities for addressing all aspects of emergencies, in particular preparedness, response and initial recovery steps", UNISDR 2009). ESCAP 2015 stresses the important role of innovative technologies for disaster assessment, mentioning the experience of the Government of India during the cyclone Hudhud damage assessments in 2014, when Geomaticsrelated technologies (GIS, GPS and remote sensing) were used to assess damage and upload information onto a satellite map using geotagging. Furthermore the National Remote Sensing Centre (NRSC) launched a crowdsourcing campaign to allow photos of damages to be uploaded by residents from their smartphone.

The present paper is mainly focused on the Rapid Mapping activities supporting the immediate emergency response phase ("the provision of emergency services and public assistance during or immediately after a disaster to save lives, reduce health impacts, ensure public safety and meet the basic subsistence needs of the people affected", UNISDR 2009), with the goal to define a generic operational workflow describing the Geomatics role in each main operational step. Geomatics plays a very important role in these phases through Emergency Mapping (defined as "creation of maps, geo-information products and spatial analyses dedicated to providing situational awareness emergency management and immediate crisis information for response by means of extraction of reference (preevent) and crisis (post-event) geographic information/data”, IWG-SEM 2014), and particularly in the response phase by means of Rapid Mapping activities, "the on-demand and fast provision (within hours or days) of geospatial information in support of emergency management activities immediately following an emergency event" (source: European Union, http://emergency.coper nicus.eu/mapping/ems/service-overview). Rapid Mapping was indeed largely adopted to support the emergency management activities related to the major disasters occurred in last years, including the aforementioned cyclone Hayan in the Philippines, as clearly demonstrated by the numbers of maps produced by the main actors in the Emergency Mapping domain for that specific emergency. ${ }^{1}$ The increasing adoption of Rapid Mapping as standard approach to support emergency management activities (exploiting mainly satellite imagery as main data source as described in Sect. 3.2), is also demonstrated by the existence of the International Working Group on Satellite Emergency Mapping (IWG-SEM, http://www.iwg-sem. org), a voluntary group of organizations involved in satellite based Emergency Mapping. It was founded to improve cooperation, communication and professional standards among the global network of satellite based Emergency Mapping providers.

The aim of the paper is therefore to highlight the main advantages of introducing Geomatics techniques in standard operational procedures, also according to the authors background in the Geomatics domain (and current active roles in national and international related initiatives, for example, the ISPRS WG VIII/1 on Disaster and Risk Reduction) and long experience in providing an operational 24/7 Rapid Mapping service since 2007 (both in the framework of the European Commission Emergency Management Service-Mapping. http://emergency.coperni cus.eu and in supporting the United Nation World Food Programme response activities). It is therefore not intended to be neither an operational handbook nor a step-by-step guide for generating Rapid Mapping products, but a critical review of the current Geomatics role in the aforementioned domain and a discussion on potential Geomatics-related research opportunities.

\section{An operational rapid mapping workflow}

In the emergency response phase, authorities in charge for disaster management activities (e.g., civil protection) at

\footnotetext{
${ }^{1}$ Copernicus Emergency Management Service-Mapping (http:// emergency.copernicus.eu/mapping/list-of-components/EMSR058), Center for Satellite Based Crisis Information (ZKI) (http://www.zki. dlr.de/article/2592), Sertit (http://sertit.u-strasbg.fr/RMS/action. php?id=9564454101), Unosat/UNITAR http://www.unitar.org/uno sat/maps/PHL.
} 
local (e.g., responders in the fields, search and rescue teams), national (e.g., civil protection national authorities, humanitarian organization regional offices) and international level (humanitarian organization or United Nations agency headquarters), need timely information about the location and extent of the event, the overall level of damage (with a focus on critical infrastructures) and the estimated number of affected people. The aforementioned information are generally represented in form of maps (and underlying datasets), infographics or reports aimed at effectively summarizing the event impact. This is clearly demonstrated by the main type of data stored in ReliefWeb (http://reliefweb.int/), a specialized digital service of the United Nations Office for the Coordination of $\mathrm{Hu}$ manitarian Affairs (OCHA) mainly aimed at collecting, delivering and enabling reliable and timely humanitarian information on global crises and disasters. For instance, in the case of the typhoon Haiyan, during the response phase in November 2013 (first 20 days after the event), a total of 278 documents were made available in ReliefWeb, specifically 235 maps and 43 infographics (similar figures also highlight a possible risk of information overflow, especially in occasion of major events where several entities are involved in the Rapid Mapping activities and several different products communicating very similar information may lead to confusion for the users on the most updated/reliable information to be used).

Rapid Mapping aims at providing this type of information to the aforementioned end users in the shortest timeframe possible, generally exploiting satellite sensors, properly tasked according to the event type, as main information source (see Sect. 3.2 for a detailed analysis). Obviously also main topographic features depicting the situation before the event should be available (e.g., as hydrography, settlements, transportation network, utilities, land cover), to geographically contextualize the event and to correctly estimate its impact. Furthermore ancillary information such as the affected population should be provided, exploiting available census datasets.

To minimize the time required to provide the post-event information to the end users, the provision of Rapid Mapping products should be based on consolidated operational workflows: a simplified workflow, highlighting the main processing steps generally adopted in the context of Rapid Mapping according to the authors experience and the operational setup of Rapid Mapping services, is shown in Fig. 1. It has to be stressed that the proposed workflow has to be intended as a general operational mapping setup supporting the emergency response phase, to highlight the main relevant operational steps and the related advantages or current limitations, in line with the paper aim. It is not related neither to a specific disaster type nor to a specific emergency management framework: different emergency management regulation exists at different level, also depending on the type of organization involved in the emergency response phase, therefore, tailored workflows may be proposed to better fit specific and local regulations and requirements.

Geomatics role in a general Rapid Mapping workflow is mainly aimed at efficiently and rapidly combining available geospatial datasets, made available by authoritative National Mapping Cadastre Agencies (NMCA) or by Collaborative Mapping initiatives like OpenStreetMap (OSM), with post-event data, to produce and disseminate value-added information exploiting affordable and operational Spatial Data Infrastructures (SDI).

The analysis of the workflow allows to highlight the following critical issues. First, to be able to determine the impact of the event over an affected area, not only the availability of post-event imagery is a crucial point, but also the availability of reference geospatial datasets depicting the situation before the event $\left(\mathrm{T}_{-1}\right)$ is essential to improve the analysis accuracy and completeness. Second, the effectiveness of the overall procedure is strictly linked to how rapidly the service products are made available to the users, considering that the main aim is to deliver postevent information few hours after the availability of the post-event imagery. The milestones of the process are related to: $\mathrm{T}_{-1}$, the time frame when pre-event reference/ thematic data should already be available; $\mathrm{T}_{0}$, the occurrence of the event; $T_{1}$, the time frame related to the availability of updated post-event data (usually a satellite image acquired specifically to respond to the emergency); $\mathrm{T}_{2}$, the time frame required to extract post-event crisis information as well as added-value information, $\mathrm{T}_{3}$, the time frame when post-event crisis information (both vector and traditional map outputs) are made available to the user community.

Taking the overall production time into account, involving all the processing steps highlighted in Fig. 1, the time constraint is very demanding and challenging, and only a fine-tuned customized operational methodology can grant the effectiveness of the overall methodology. It has to be highlighted that currently the bottleneck of similar services is the time required to get usable post-event satellite imagery, considering all the related operational steps (acquisition request, satellite triggering, imagery acquisition, data download to the receiving station, data preprocessing and final delivery). In the framework of the Copernicus Emergency Management Mapping service, this phase takes approx. $39 \mathrm{~h}$ while the remaining steps (imagery processing and map production) are limited to approx. $5.5 \mathrm{~h}$ (mainly thanks to the adoption of proper and standardized Geomatics techniques), according to the average values based on more than 30 service activations (Priolo 2014). 


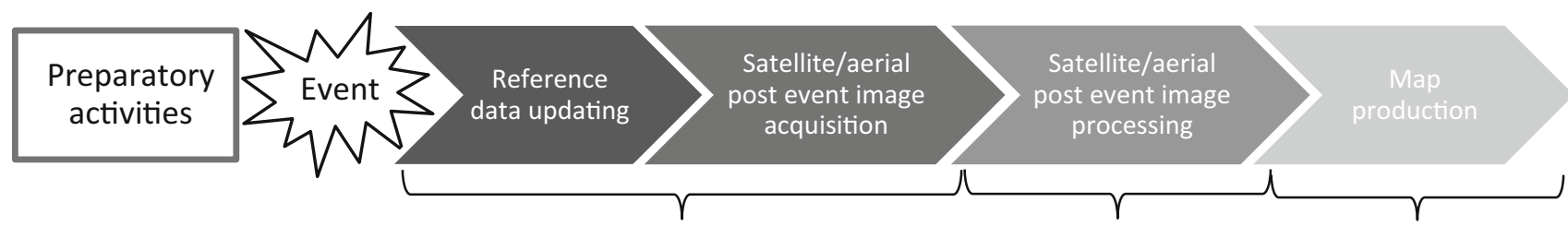
$T_{-1}$
$\mathrm{T}_{0}$
$\mathrm{T}_{1}$
$\mathrm{T}_{2}$
$\mathrm{T}_{3}$

Reference data covering the

affected area before the event

$\mathrm{T}_{-1}$

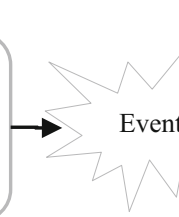

ETL procedures (if necessary)

- Data extraction

- Data transformation (model mapping)

- Data loading

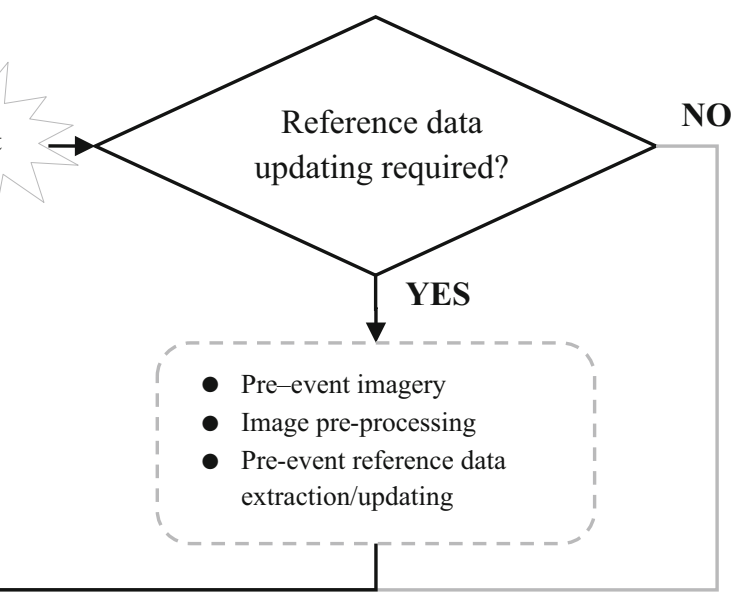

Up-to-date reference data covering the affected area before the event

- Image pre-processing (geocoding, orthorectification, coregistration)

- Extraction of Post-event information

Satellite/aerial post-event acquisition $\mathrm{T}_{1}$

- Extraction of value-added information

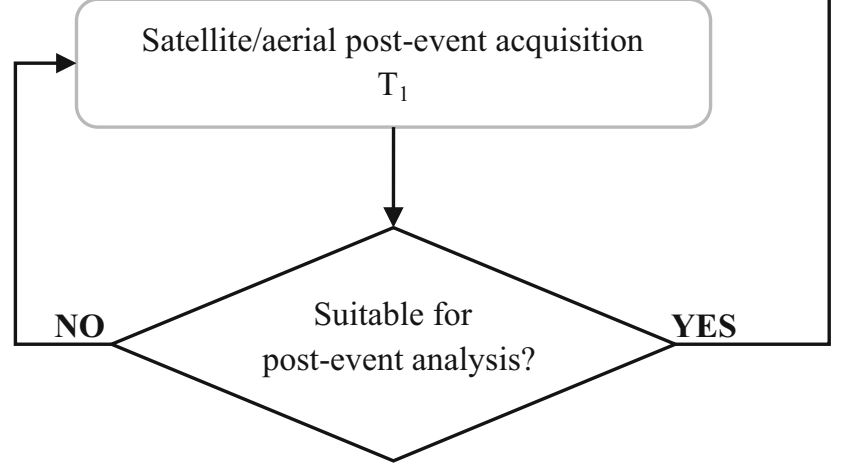

$\mathbf{I}$

Post-event emergency information covering the affected area

$\mathrm{T}_{2}$

Map dissemination

$\mathrm{T}_{3}$

Fig. 1 Simplified Rapid Mapping general flow-chart highlighting the main processing steps and the activity timeline

The analysis of the workflow highlights that Geomatics techniques are exploited in all the processing steps, allowing the service constraints (especially in terms of limiting as much as possible the time span from $T_{1}$ to $T_{3}$ ) to be fulfilled, i.e.:

- provision of data quality information and delivery of replicable products;

- provision of standard metadata;

- use of consistent data models, for the implementation of a data interoperability strategy allowing the re-use (even partial) of the data;

- adoption of a multi-scale approach, able to grant consistency among datasets covering larger areas and analysis at a higher level of detail focused on ad-hoc detail areas;

- adoption of a dissemination strategy, to maximize the access to the information from the user community.

Furthermore, Geomatics may play a crucial role in the future developments, for example.:

- the automation of feature extraction procedures (especially for crisis layers) to avoid possible subjectivity and heterogeneity in the analysis;

- the generalization of the data processing algorithms to different source of data, with a main focus on SAR data (which may be the only accessible in case of persistent cloud coverage). 
As already described in other publications of the authors (Boccardo and Giulio Tonolo 2015; Boccardo 2013; Boccardo and Rinaudo 2011; Boccardo and Giulio Tonolo 2008), different International initiatives are already operational in the field of Emergency Mapping, as clearly demonstrated by the existence of the International Working Group on Satellite Emergency Mapping (IWG-SEM, http:// www.iwg-sem/.org). Unfortunately, not all the existing mechanisms cover all the operational steps involved in the Emergency Mapping workflow and some of these initiatives are focused only on specific geographical areas. Furthermore most of the aforementioned points, for example, product specifications, description of data models, metadata availability, adopted methodologies, are often not evident or unknown to the final user.

\section{Geomatics and rapid mapping}

Different techniques within the Geomatics domain could be exploited in a Rapid Mapping workflow; i.e., GIS/Digital Mapping (for reference data management), Remote Sensing and Photogrammetry (for managing imageries acquired by satellite/aerial platforms, including unmanned platforms), SDI (for data modeling and management, spatial analysis, layouts customization and data dissemination).

The Geomatics fields related to the processing steps in the flowchart will be described in the following paragraphs, while potential future developments and research opportunities will be addressed in Sect. 4. The following paragraphs are not aimed at being a detailed and complete operational guide on how to apply specific tools, but at highlighting the main advantages that could be granted by the adoption of proper Geomatics techniques.

\subsection{Reference data}

In the specific context of Emergency Mapping, reference data are intended as selected topographic features to be used to contextualize the event, to produce comprehensive and easy-to-use map products and to provide value-added information on damages (Voigt et al. 2007). The systematic availability and the access to accurate and up-to-date spatial data at a suitable map scale are crucial issues, especially considering that Emergency Mapping has a global coverage requirement. Geo-information for crisis management should be retrieved directly from the data provider, to optimize data acquisition and management processes and to access the latest available update of the data (Diehl et al. 2006; Aalders and Moellering 2001).

The benefits of interoperable Spatial Data Infrastructures (SDI) for data access and dissemination in the framework of disaster response are already demonstrated.
Well documented data structures and efficient data transfer services are fundamental conditions allowing the integration of information coming from different sources, especially in Rapid Mapping workflows where the time constraint is a critical factor (Mansourian et al. 2006; Diehl et al. 2006). International initiatives, such as the United Nations Spatial Data Infrastructure (UNSDI), the United Nations initiative on Global Geospatial Information Management (UN-GGIM), the Global Earth Observation System of Systems (GEOSS) and the Infrastructure for Spatial Information in the European Community (INSPIRE), aimed at ensuring that the spatial data infrastructures are compatible and usable in a multidisciplinary community and in a transboundary context, have the role to provide the organizational and legal framework required for an effective SDI implementation.

Official and authoritative reference datasets availability is an additional key element, not simply due to the intrinsic higher quality associated to data provided by official national and international authorities, normally generated following well-established acquisition procedures and verified by means of severe testing procedures. Even more significant is the fact that generally Rapid Mapping final users are entities (e.g., national and transnational civil protection authorities) that are used to work with official and authoritative reference data: therefore, value-added analysis and products based on those common datasets produce results that can be immediately integrated in operational procedures and used to carry out additional inhouse analyses tailored to the user needs. As an example, an earthquake damage assessment at single building level made on official cadastral data would streamline the process of identifying the affected owners and estimating the related economic loss.

Despite the fact that currently no major technical constraint prevent from establishing SDI solutions, a systematic access to official and authoritative reference datasets cannot yet be considered an operational step within a standard Rapid Mapping production workflow. In regions where SDI culture is more established, operational data access through web-based services can be considered on a case-by-case basis, after an in-depth evaluation of legal constraints and national cartographic service organization (e.g., fragmented or overlapping responsibilities in reference data acquisition and maintenance), elements that may have a negative impact on the effectiveness of this approach. In other areas of the world, where the lack of financial resources and infrastructure limitations (i.e., internet network availability and performances) are common situations, a generalized access to reference data through SDI solution is currently not an option.

International organizations, particularly those involved in the humanitarian aid, risk reduction and recovery sectors 
(e.g., UN agencies and the World Bank) may partially fill the gap in the availability of reference data in emerging countries. To efficiently accomplish their mandate, a big effort is normally spent in collecting all data available at local level, in acquiring new data and in setting up SDI solutions. UN OCHA, one of the leaders of UN Emergency Telecommunications UN cluster, has a series of on-going initiatives in the information management field, such as Reliefweb (http://reliefweb.int/about), HumanitarianResponse.info (https://www.humanitarianresponse.info/) and GDACS (http://www.gdacs.org/). UN WFP, the larger humanitarian agency in the World, is currently building its own SDI platform, populated by data directly harvested in more than 70 different countries and directly acquired through the Logistic Cluster operations (http://www. logcluster.org/). The Global Facility for Disaster Reduction and Recovery (GFDRR), a global partnership managed by the World Bank and funded by 25 donor partners, established the Open Data for Resilience Initiative (OpenDRI), aiming to reduce the impact of disasters by empowering decisions makers with better information and the tools to support their decisions: GeoNode (http://geonode.org/), an open-source geospatial data management platform, is one of the tangible products of this initiative.

Public domain global datasets represent an alternative to official and authoritative local reference datasets. VMap (NIMA 2000), a comprehensive vector-based collection of reference data layers, is a solution for small scale analysis (VMAP level 0, the only level released into public domain, has a reference scale equal to $1: 1.000 .000$ ) and in case there is no need for recently updated data. For analysis at larger map scales and requiring more up-to-date reference data, Collaborative Mapping initiatives are more and more considered as an effective solution: the disadvantage of not generating official data is often overcome by the fact that, at least in some region of the world, Collaborative Mapping initiatives are capable to provide recently updated information. OpenStreetMap (OSM, https://www.open streetmap.org) is a collaborative project to create a free editable map of the world: currently, OSM counts on over 1.6 million registered users for generating comprehensive geographic reference data from different sources (e.g., manual survey, GPS devices, aerial and satellite imagery); additionally, the Humanitarian OpenStreetMap Team (HOT, http://hot.openstreetmap.org/) applies the principles of open source and open data sharing for humanitarian response and economic development, focusing data acquisition activities in areas currently affected by humanitarian emergencies or that are considered particularly vulnerable. Similarly, Wikimapia (http://wikimapia.org/) aims to create and maintain a free, complete, multilingual and up-to-date map of the whole world. GeoNames is a geographical database containing over 10.000 .000 geographical names, available and accessible through various web services. The adoption of Collaborative Mapping initiatives opens the issue of developing quality evaluation protocols and tools for those data, generated without standard cartographic production workflows (Ajmar et al. 2011b).

\subsection{Remote sensing}

In the framework of Rapid Mapping, different options can be considered for the acquisition (i.e., platforms and sensors) and processing of post-event remotely sensed imagery depicting the situation after the event (Boccardo and Giulio Tonolo 2015). Several factors should be considered to identify the best option, for example., the type of disaster to be monitored (and consequently the specific crisis information to be provided to the end users), the extent of the affected areas, the required level of detail of the analysis (i.e., the nominal map scale). Furthermore end-user requirements should be always taken into account, to potentially tailor the analysis to specific needs.

As far as Remote Sensing platforms are concerned, generally satellite platforms are exploited, allowing large areas, potentially with limited or no accessibility due to the event impact, to be sensed and, if required, monitored. Furthermore, modern agile satellites can be triggered in a very short time allowing, in the best case scenario, to have images covering the areas of interest few hours after the request. The actual timeliness of a satellite acquisition depends on several technical factors such as the affected area local time, the distribution of ground receiving station, the local weather conditions, possible conflict with other planned tasks and the requirements of the user in terms of imagery technical features (e.g., limited off-nadir angle for optical sensors). Aerial platforms can be adopted to grant the acquisition of photogrammetric imagery characterized by a very high level of detail, allowing to derive not only orthoimagery but also ancillary datasets as Digital Surface Models (DSM) or 3D features. Recently, also Remotely Piloted Aircraft Systems (RPAS) or UAV (Unmanned Aerial Vehicles) are being tested in Emergency Mapping contexts, both in demonstration exercises (Rester et al. 2013) and for Post Disaster Needs Assessment (PDNA) purposes, as during the recent Balkan Floods in May 2014 (http://irevolution.net/2014/07/07/humanitarian-uav-mis sions-during-balkan-floods/).

As far as the sensor type is concerned, radar SAR sensors are generally exploited when the persistency of cloud cover make optical data unusable, when it is required to extract very accurate information on ground displacements (e.g., geophysical events) or object movements over the time (e.g., lava flows), or when it is necessary to acquire information with an high-revisiting time (exploiting the 
nighttime passes). Optical sensors are the preferred choice to carry out damage assessments at a very high level of detail (e.g., building damage assessment) or when multispectral information is required (e.g., burnt scars classification), and they are obviously the only choice when a visual interpretation approach has to be adopted for the post-event analysis, due to the lack of robust and efficient automatic classification techniques.

Concerning the spatial resolution, both optical and radar sensors can nowadays acquire very high spatial resolution (VHR) imagery (with a ground sample distance up to $0.3 \mathrm{~m}$ for optical sensors). This type of imagery is characterized by a very high level of detail with a high positional accuracy (using standard post-processing algorithm as the rational polynomial function approach) and a high semantic content, crucial features when assessing the impact of a disaster. VHR optical imagery in general has been available for almost a decade and proven to be useful in damage assessment in a number of natural disasters (Ehrlich et al. 2009). On the contrary, medium/low spatial resolution imagery is characterized by a larger footprint and a wider spectral resolution, features that may be crucial when monitoring regional-wide disasters (e.g., monsoonal flooding in tropical regions).

Temporal resolution is a critical factor when monitoring disaster evolution over the time (e.g., flood wave or fire propagation): the availability of constellation of earth observation satellites (even virtual constellations made of different types of satellites operated by the same institution) as well as the increasing number of satellite data providers, drastically decrease the satellite revisiting time (increasing the probability of a successful acquisition).

Lastly, the licensing policy of the data is another feature that has to be carefully considered, both in terms of imagery costs (ranging from public-domain data generally accessible through the web or commercial imagery that should be purchased through reseller companies) and potential dissemination constraints which may prevent final user to have direct access to raw imagery.

Most of the above mentioned factors are relevant also referring to pre-event remotely sensed imagery, to be used to update reference data, in case that is needed and where no other appropriate data sources (i.e., national official imagery) are available.

\subsection{SDI and process automation}

Rapid Mapping requires a high level of automation in value-added information generation (e.g., estimation of the affected population or of potential damages to infrastructures) and map production: automation streamlines all the analysis and mapping processes, minimizing the risk of errors and shrinking production times.
High levels of automation require to operate with standard and consolidated geodatabase models. With such prerequisite, it is relatively straightforward to setup analysis tools that extract information on exposed population or assets or that, combining reference data with crisis layers, derive figures on the consequences of a specific event. Similarly, standard symbolization rules and map templates allows to realize products at the same time easy to be interpreted and clearly branded. In most cases, Rapid Mapping services are requested to produce and deliver not simply raster maps but also the single vector components, allowing the final user to produce more customized analysis and maps: automatic export procedures or web services can be easily setup, providing information in their original data structure or in customized structures and formats.

Metadata contains critical information not only to grant a proper integration of information from different sources but also to allow the delivered products (in the format of either maps or single vector files) to be fully understood by the end users. Geospatial metadata standards, such as ISO 19115-2, provide all indication to efficiently organize metadata relevant to many geospatial data sets, including raster imagery, GPS, monitor stations, instruments, etc.

From the operational perspective, there are currently commercial and Open Source solutions covering all major component of an SDI architecture: geodatabase server, web server and GIS application. The adoption of a particular solution is function of the specific needs and available resources.

\section{Rapid Mapping main operational steps: consolidated approaches and research opportunities}

The simplified Rapid Mapping workflow shown in Fig. 1 (representing the average operational scenario, not the best neither the worst situation), has been analyzed to identify the specific processing steps that can be further improved or where open issues are still present.

The most commonly adopted technologies, the main open issues and the potential research topics that-according to the authors-should be considered for improving the performances of Rapid Mapping services, have been therefore summarized in Table 1 and discussed in chapter 4 subsections.

Once again it is stressed that the analyzed main operational steps refer to a general Rapid Mapping workflow, which is in line with the paper aim to highlight and discuss the current and futurel role of Geomatics. It is not to be intended neither related to a specific disaster type nor focused on a specific emergency management framework, which may require tailored workflows defined on a caseby-case basis. 
Table 1 Rapid Mapping main operational steps: open issues and potential research topics

\begin{tabular}{|c|c|c|c|}
\hline Processing step & Methodological topic & Current solution & Open issues/potential research topics \\
\hline Satellite data tasking & $\begin{array}{l}\text { Sensors and platforms from } \\
\text { air and space }\end{array}$ & Priority orders to data providers & $\begin{array}{l}\text { Integration of weather forecast in satellite } \\
\text { tasking to avoid high cloud coverage in } \\
\text { tasked imagery } \\
\text { Integration of microsatellite and RPAS to } \\
\text { complement post-event data acquisition }\end{array}$ \\
\hline $\begin{array}{l}\text { Extraction, } \\
\text { transformation and } \\
\text { loading (ETL) } \\
\text { procedures }\end{array}$ & Geospatial databases & $\begin{array}{l}\text { Ad-hoc ETL procedures (data model } \\
\text { mapping) }\end{array}$ & $\begin{array}{l}\text { Operational exploitation of existing standard } \\
\text { transboundary SDI initiatives (e.g., UNSDI, } \\
\text { GEOSS, INSPIRE) }\end{array}$ \\
\hline Imagery pre-processing & $\begin{array}{l}\text { Sensor and image } \\
\text { orientation }\end{array}$ & $\begin{array}{l}\text { RPC or rigorous orientation models } \\
\text { (with GCP if available), image } \\
\text { coregistration }\end{array}$ & $\begin{array}{l}\text { Improvement of global DEM resolution and } \\
\text { vertical accuracy } \\
\text { Improvement of (automated) coregistration } \\
\text { procedures }\end{array}$ \\
\hline \multirow[t]{2}{*}{$\begin{array}{l}\text { Pre-event reference data } \\
\text { extraction/updating } \\
\text { and }\end{array}$} & Geospatial databases & $\begin{array}{l}\text { Exploitation of NMCA datasets and } \\
\text { collaborative mapping global } \\
\text { initiatives (e.g., OSM) }\end{array}$ & $\begin{array}{l}\text { Removal of data access (e.g., license, } \\
\text { infrastructure) barriers } \\
\text { Development and implementation of data } \\
\text { quality protocols and tools }\end{array}$ \\
\hline & & $\begin{array}{l}\text { Computer aided photo interpretation } \\
\text { (CAPI) }\end{array}$ & $\begin{array}{l}\text { Development/improvement of semi- } \\
\text { automated features extraction procedures } \\
\text { Ad-hoc collaborative mapping campaign }\end{array}$ \\
\hline $\begin{array}{l}\text { Post-event information } \\
\text { extraction }\end{array}$ & $\begin{array}{l}\text { Image } \\
\text { interpretation/classification }\end{array}$ & $\begin{array}{l}\text { Semi-automated classification and/or } \\
\text { CAPI }\end{array}$ & $\begin{array}{l}\text { Improvement of thematic accuracy and } \\
\text { automation level } \\
\text { Adoption of collaborative approach to CAPI } \\
\text { Exploitation of 3D data }\end{array}$ \\
\hline $\begin{array}{l}\text { Value-added } \\
\text { information extraction } \\
\text { from ancillary data }\end{array}$ & $\begin{array}{l}\text { Semantic and geometric } \\
\text { integration }\end{array}$ & $\begin{array}{l}\text { Tools for summary statistics } \\
\text { generation, including affected } \\
\text { population }\end{array}$ & $\begin{array}{l}\text { Generation/integration/updating of ancillary } \\
\text { global datasets }\end{array}$ \\
\hline Map dissemination & Digital cartography & $\begin{array}{l}\text { (s)FTP/HTTP(s) protocols } \\
\text { Ad-hoc web portal } \\
\text { Web Services (i.e., WMS, WFS, } \\
\text { WCS) }\end{array}$ & Exploitation of dynamic live maps \\
\hline
\end{tabular}

\subsection{Satellite data tasking}

Quality imagery is one of the prerequisite for Emergency Mapping: in addition, imagery provided in the shortest time possible is crucial for Rapid Mapping. One of the main open issue related to post-event imagery is the risk of persistent cloud coverage (especially relevant for hydrometeorological events or in specific geographical areas), that prevent from the use of optical imagery for post-event analysis.

An integration of accurate weather forecast technologies in satellite tasking would allow to avoid unnecessary acquisitions (with clear impact on costs) or to redirect the choice to SAR sensors, currently mainly adopted only for specific applications.

The recent availability of microsatellite (characterized by a size up to 20 times smaller than traditional platforms and optimized efficiency) allows constellations made of several platforms to be planned in the near future. An increased number of satellites will allow to boost the time required to deliver the acquired data (imagery download in 20 min of collection, http://www.skybox.com/uploads/11/ 01/skynodedatasheet1-6-14.pdf), reducing the triggering time (up to $10 \mathrm{~min}$ before the satellite flies over) as well as increasing the acquisition possibilities (5-7 times per day with the full 24 satellites constellation, http://apogeospa tial.com/apogeo-qa-skybox-imaging/).

Lastly, in case the required level of detail cannot be fulfilled by standard satellite/aerial platform, the adoption and consolidation of RPAS solutions should be encouraged.

\subsection{ETL procedures}

To exploit available reference datasets in a Rapid Mapping context, procedures for data extraction, transformation and loading into the ad-hoc databases adopted for emergency purposes are commonly used.

While loading can be performed by completely automated procedures, data extraction and transformation would need ad-hoc procedures, depending on the required 
reference datasets. As mentioned in Sect. 3.1, the adoption (and implementation) of standard transboundary models would lead to the definition of a limited number of standard extraction and transformation procedures, with clear advantages in terms of efficiency in Rapid Mapping. In this context, the development of on-the-fly WFS data model mapping applications may be considered the best trade in terms of timing and effort.

\subsection{Imagery pre-processing}

To carry out multi-temporal analyses, the most common approach adopted to extract post-event crisis information, the available pre and post-event imagery requires to be geometrically corrected, using orthocorrection algorithms based on RPC or rigorous orientation models (with GCP if available), and most importantly, coregistered.

Giving the global nature of Rapid Mapping activities, global DEM datasets are often the only available source of elevation information required for the orthocorrection step. The limited spatial resolution and height accuracy may decrease the overall geometric accuracy of the resulting dataset. Therefore, actions focused on the improvement/integration of available datasets, as well as on the creation of new regional/global dataset with a higher elevation accuracy and spatial resolution, should be encouraged.

As far the coregistration process is concerned, one of the most challenging issue is the development of semi-automated tools for the coregistration of optical and radar imagery.

\subsection{Pre-event reference data extraction/updating}

Official and authoritative sources of reference data produced and maintained by NMCA are clearly the first option: but even in regions with consolidated experiences in geographic data sharing mechanisms, licensing and/or infrastructural issues remain barriers which may prevent access to the data.

When NMCA reference datasets are not available, datasets produced by collaborative mapping initiatives as OpenStreetMap are generally exploited, but a thorough analysis is required to assess the quality level, especially referring to completeness and updating. Missing, incorrect or not updated features are mainly edited by means of Computer Aided Photo Interpretation (CAPI) on the available orthoimagery, with an obvious impact on the required effort, especially in terms of timing. To cope with the aforementioned issue, the development of ad-hoc procedures for the semi-automated extraction of reference feature should be encouraged, including tools supporting manual feature extraction during CAPI.
Furthermore, in case of Emergency Mapping activities focused on areas with no detailed reference datasets available, ad-hoc collaborative mapping campaign should be encouraged, exploiting initiatives like the Humanitarian OpenStreetMap Team (HOT-OSM, http://hot.open streetmap.org/), with the goal to drastically reduce the time effort for the reference data extraction, focusing on the post-event analysis.

\subsection{Post-event information extraction}

Currently post-event information extraction from remote sensed imagery is performed by means of automatic and/or semi-automatic procedures for limited types of events (e.g., in case of floods, as described in Ajmar et al. 2008) as well as through CAPI (e.g., for earthquake damage assessment, as highlighted in Ajmar et al. 2011a).

Improved automatic procedures for the extraction of crisis information should be foreseen to reduce the production and the human interpretation times. A more extensive use of radar technology should be considered, for example., to extend its consolidated operational use also for events different from floods (such as landslide, earthquakes or fires), with the goal to increase the post-event acquisition possibilities exploiting the all-weather capabilities of radar sensors.

Furthermore, technology improvements in the satellite earth observation domain over the last years may play a key-role in consolidating the use of satellite imagery for emergency response purposes as well as in making new application possible. Currently (as of October 2014) some cutting-edge technology seems to be very promising, specifically:

- video capabilities: SkySat-1 (an optical microsatellite launched at the end of 2013) is capable of acquiring not only sub-meter imagery but even high definition panchromatic videos up to $90 \mathrm{~s}$, a feature that was never available before;

- improved spatial resolution: new platforms, already or very soon operational (i.e., WorldView-3) are characterized by a spatial resolution of the panchromatic band up to $0.30 \mathrm{~m}$ which will increase the level of detail of the imagery and, consequently, the thematic accuracy of the extracted value-added information.

Nevertheless, the increase of satellite data availability and acquisition capacity (in terms of both coverage and revisiting time) poses new challenges in the Rapid Mapping domain, especially in terms of availability of resources required to process and analyze a potentially huge amount of data in a suitable time frame. Recent initiatives like TomNod (http://www.tomnod.com/) are a possible solution, since they exploit the power of crowdsourcing 
simply to identify objects and places in satellite images to solve real-world problems which are most often related to disaster response.

\subsection{Value-added information extraction from ancillary data}

Value-added crisis information accuracy is intrinsically related to the thematic accuracy of available ancillary information such as land cover and census datasets. As already highlighted while discussing the research opportunities related to elevation data, the improvement/ integration of available ancillary datasets, as well as the creation of new regional/global ancillary dataset with a higher thematic accuracy, should be encouraged.

\subsection{Map dissemination}

Data dissemination is an important part of the Rapid Mapping workflow, due to the need for the end user to access the post-event information as soon as available. The use of standard static mapping products, made available for download in ad-hoc Web portals through (s)FTP or HTTP(s) protocols is a consolidated dissemination strategy.

To complement the Emergency Mapping workflow, the use of standard Web services enabling users to access crisis information in near-real time (as soon as generated and quality checked) should be encouraged.

\section{Conclusions}

The present contribution was mainly focused on the Rapid Mapping activities supporting the immediate Emergency Response phase. A general Rapid Mapping operational workflow has been proposed in Sect. 2, highlighting the limits of a "general" framework, which was necessary to identify the main operational steps and to describe the Geomatics-related fields which are currently exploited in these domains (Sect. 3). It is evident that one of the main goal of a Rapid Mapping service is shortening as much as possible the time required to deliver accurate and updated post-event information to the final users. While most of the analyzed Geomatics fields are already mature enough to provide an effective operational support to Rapid Mapping, two main current bottlenecks have been highlighted:

- first, the lack of a wide implementation of existing interoperable and transboundary SDI initiatives, to grant an operational access to authoritative datasets and a rapid integration of geographical information provided by different sources;
- second, the time span required to task, acquire and deliver new satellite acquisitions, which are currently taking most of the total time spent from the initial Rapid Mapping service activation to the delivery of the final outputs to the users: laser link download technology recently tested by the European Space Agency is a technical solution which decreases this time span, allowing large volumes of remotely sensed data to be readily available (ESA 2014).

The most commonly adopted Geomatics techniques exploited in the main Rapid Mapping operational steps have been synthetically reviewed in Sect. 4, with a focus on the major open issues and the potential research topics that should be considered for improving the performances of Emergency Mapping services. The following list of research areas are the ones considered by the authors of particular interest for the Geomatics community:

- investigation of the possible role of recent technologies (e.g., RPAS or UAV) to complement satellite imagery as post-event data source. International initiatives like the Humanitarian UAV Network (http://uaviators.org/) are trying to bridge humanitarian and UAV communities internationally with the goal to facilitate information sharing, coordination and operational safety in support of a broad range of humanitarian efforts. The European Commission is already assessing the potential role of UAVs, using the collected imagery "as an alternative and/or complementary source of post-event imagery in emergency situations and in a rapid response and mapping context" (Copernicus 2015);

- developing robust semi-automated procedures for the extraction of post-event crisis information, to reduce the need for CAPI (a time-consuming activity) and with the goal to ensure a thematic accuracy comparable to the one achievable by means of photointerpretation techniques, or at least to allow the use of similar outputs as input for a more rapid visual check by image analyst specialist. The role of interesting new technologies (e.g., videos acquired by satellite platforms) should be further investigated and assessed, especially in terms of potential new type of information that can be extracted (e.g., population movements in a security context);

- systematically evaluating, in terms of both efficiency and thematic accuracy, a potential structured role of collaborative mapping and crowdsourcing initiatives (e.g., HOT-OSM, TomNod) in analyzing post-event imagery. Interesting recent activities are also involving the volunteer community in "off-line" (e.g., not during an emergency response activity) analyses to be used as input and/or ground-truth for supervised classification algorithms. 
Open Access This article is distributed under the terms of the Creative Commons Attribution License which permits any use, distribution, and reproduction in any medium, provided the original author(s) and the source are credited.

\section{References}

Aalders HJGL, Moellering H (2001) Spatial data infrastructure. In: Proceedings of the 20th international cartographic conference. Beijing, China (pp. 2234-2244)

Adikari Y, Yoshitani J (2009) Global trend in water-related disasters: an insight for policymakers. UNESCO, 7 place de Fontenoy, 75352 Paris, France ISBN 978-92-3-104109-9. http://www. unwater.org/downloads/181793E.pdf. Accessed 19 Feb 2015

Ajmar A, Boccardo P, Disabato F, Giulio Tonolo F, Perez F, Sartori G (2008) Early impact procedures for flood events February 2007 Mozambique flood. Rivista italiana di Telerilevamento, 40(3):65-77. doi: 10.5721/ItJRS20084036. http://www.aitjour nal.com/recdStat.aspx $?$ ID $=272 \&$ mode $=1$. Accessed 13 Oct 2014

Ajmar A, Boccardo P, Giulio Tonolo F (2011a) Earthquake damage assessment based on remote sensing data. The Haiti case study. Italian Journal of Remote Sensing 43(2):123-128. doi: 10.5721/ ItJRS20114329. http://www.aitjournal.com/recdStat.aspx?ID= $227 \&$ mode $=1$. Accessed 13 October 2014

Ajmar A, Balbo S, Facello A, Perez F, Steffenino S, Vigna R (2011b) Geospatial reference data access for emergency response: data inventory and quality assessment. GeoInformation for Disaster Management (GI4DM), Antalya (Turkey), 3-8 May 2011

Boccardo P (2013) New perspectives in Emergency Mapping. Eur J Remote Sens 46:571-582. doi:10.5721/EuJRS20134633. http:// www.aitjournal.com/recdStat.aspx? $? \mathrm{ID}=849 \&$ mode $=1$. Accessed 13 Oct 2014

Boccardo P, Giulio Tonolo F (2008) Natural disaster management: Activities in support of the UN system. In: Li Zhilin, Chen Jun, Baltsavias Emmanuel (eds) Advances in Photogrammetry, Remote Sensing and Spatial Information Sciences: 2008 ISPRS Congress Book. CRC Press, Boca Raton, pp 385-396. ISBN 9780415478052

Boccardo P, Giulio Tonolo F (2015) Remote Sensing Role in Emergency Mapping for Disaster Response in Engineering Geology for Society and Territory, vol 5. Springer International Publishing, pp 17-24. doi:10.1007/978-3-319-09048-1_3, ISBN: 978-3-319-09047-4

Boccardo P, Rinaudo F (2011) Gi4DM: Geo Information for Disaster Management, Torino, February 2010. Appl Geomat 3(2):59-60. doi:10.1007/s12518-011-0051-3

Copernicus Emergency Management Service-Mapping. (2015) http://emergency.copernicus.eu/mapping/ems/new-phase-brief. Accessed 19 Feb 2015

Diehl S, Neuvel J, Zlatanova S, Scholten H (2006) Investigation of user requirements in the emergency response sector: the Dutch case in Second Symposium on Gi4DM, 25-26 Sep 2006, Goa, India, CD ROM, p 6

Ehrlich D, Guo HD, Molch K, Ma JW, Pesaresi M (2009) Identifying damage caused by the 2008 Wenchuan earthquake from VHR remote sensing data. Int J Digit Earth 2(4):309-326. doi:10. 1080/17538940902767401

ESA (2014) Laser link offers high-spped delivery. http://www.esa.int/ Our_Activities/Observing_the_Earth/Copernicus/Sentinel-1/ Laser_link_offers_high-speed_delivery. Accessed 19 Feb 2015

Guha-Sapir D, Hoyois Ph, Below R (2014) Annual Disaster Statistical Review 2013:The Numbers and Trends. Brussels: CRED. http:// www.cred.be/sites/default/files/ADSR_2013.pdf. Accessed 13 Oct 2014

IWG-SEM (2014) Emergency Mapping Guidelines. http://www.unspider.org/sites/default/files/IWG_SEM_EmergencyMappingGui delines_A4_v1_March2014.pdf. Accessed 26 Feb 2015

Mansourian A, Rajabifard A, Valadan Zoej MJ, Williamson I (2006) Using SDI and web-based system to facilitate disaster management. Computers Geosci 32(3). doi:10.1016/j.cageo.2005.06.017

Munich Re (2012) 2011 Natural catastrophes worldwide - Disaster profiles. http://www.preventionweb.net/files/24476_20120104 munichre.pdf. Accessed 19 Feb 2014

National Imagery and Mapping Agency (NIMA) (2000) Vector Map Level 0 (Digital Chart of the World). Bethesda, MD, http:// geoengine.nga.mil/geospatial/SW_TOOLS/NIMAMUSE/webin ter/rast_roam.html, Accessed 13 Oct 2014

Priolo A (2014) User Awareness \& Training: EMERGENCY training.Tallin, Estonia, 10 April 2014. http://www.copernicus.eu/ fileadmin/user_upload/Docs_for_UserUptake/TrainingSessions/ Tallinn/Emergency-Priolo-Core-Service-Overview-and-Demon strations.pdf. Accessed 12 Mar 2015

Rester M, Spruyt P, De Groeve T, Van Damme O, Ali A (2013) Unmanned Aerial Systems for Rapid Mapping-UASRapidMap 2013 4th JRC ECML Crisis Management Technology Workshop, Publications Office of the European Union, 978-92-7935107-5. doi:10.2788/58375. http://publications.jrc.ec.europa.eu/ repository/bitstream/111111111/30423/1/lbna26451enn.pdf. Accessed 13 Oct 2014

UNESCAP (2015) Disasters in Asia and the Pacific: 2014 Year in Review. http://www.unescap.org/sites/default/files/Year\%20In\% 20Review_Final_FullVersion.pdf. Accessed 26 Feb 2015

UNISDR (2009) Terminology on disaster risk reduction. http://www. unisdr.org/files/7817_UNISDRTerminologyEnglish.pdf. Accessed 19 Feb 2014

Voigt S, Kemper T, Riedlinger T, Kiefl R, Scholte K, Mehl H (2007) Satellite Image Analysis for Disaster and Crisis-Management Support. IEEE Trans Geosci Remote Sens 45(6). doi:10.1109/ TGRS.2007.895830. http://ieeexplore.ieee.org/stamp/stamp.jsp? $\mathrm{tp}=\&$ arnumber $=4215094 \&$ isnumber $=4215027$. Accessed 13 Oct 2014 